\title{
NON-SPECIFIC INFLAMMATORY BOWEL DISEASE
}

\author{
S Pettit, M H Irving
}

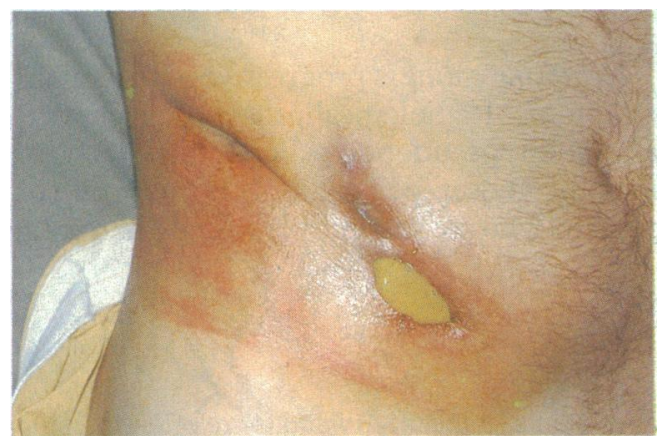

Severe manifestations of Crohn's disease with multiple enterocutaneous fistulas.

\section{Incidence}

\begin{tabular}{|c|c|c|}
\hline \multicolumn{3}{|c|}{ Incidence of Crohn's disease from } \\
\hline & $\begin{array}{c}\text { Incidence } \\
\text { (cases/ } \\
100000\end{array}$ & \\
\hline Country & population) & studied \\
\hline North east Scotland & 4.6 & $1962-8$ \\
\hline Malmö, Sweden & $6 \cdot 0$ & $1966-8$ \\
\hline United States & 4.5 & 1973 \\
\hline Cardiff, Wales & 4.9 & $1976-80$ \\
\hline Copenhagen, Denmark & 2.7 & $1970-8$ \\
\hline
\end{tabular}

\section{Pathology}
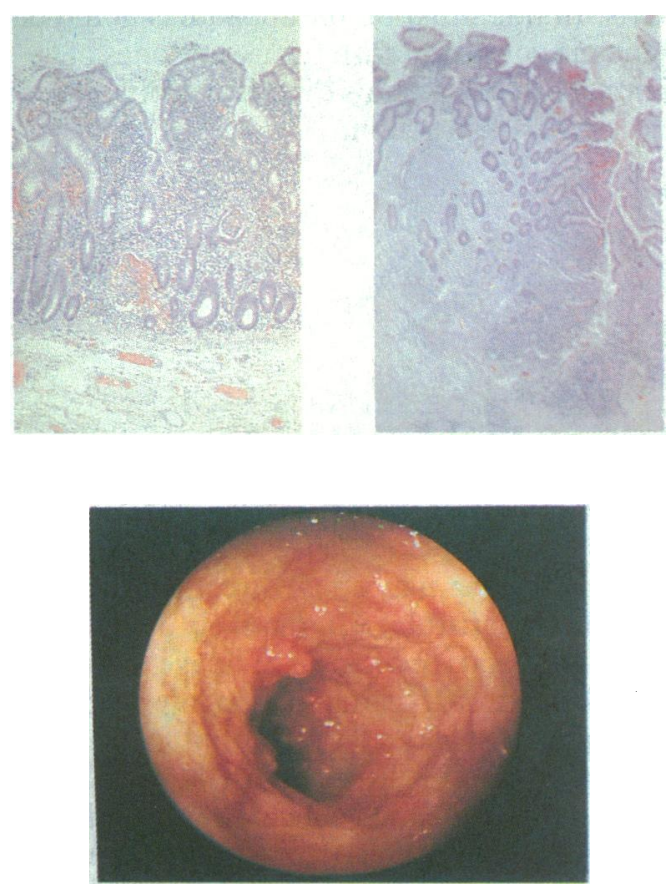

Severe ulcerative colitis viewed through a colonoscope.
Non-specific inflammatory bowel diseases are those for which there is no discernible aetiological agent. The two most important of these diseases affecting the large bowel are ulcerative colitis and Crohn's disease. Ulcerative colitis is always confined to the large bowel, although a few centimetres of the terminal ileum may be affected by "backwash" ileitis. On the other hand, Crohn's disease affects the whole of the gastrointestinal tract, although the only manifestation may be in the large bowel.

The incidence of ulcerative colitis has remained static at 26/100000 population. The incidence of Crohn's disease rose from an annual incidence of $0 \cdot 7 / 100000$ to $5 / 100000$ in the past three decades. Women are more commonly affected by Crohn's disease than men and both sexes have a peak onset in their 20s-40s with a secondary peak in their 70s. Ulcerative colitis has a similar age distribution but the sex incidence is equal.
Histological slides showing ulcerative colitis (far left) confined to the mucosa and small bowel in Crohn's disease (left) with transmural inflammation and a fissuring ulcer.
Ulcerative colitis usually begins distally as proctitis and then spreads proximally to affect the whole of the large bowel. In some cases, however, it remains confined to the rectum. Crohn's disease typically affects segments of the gastrointestinal tract with areas of diseased bowel separated by intervening areas of macroscopically normal bowel. The most common area to be affected is the terminal ileum and caecum (ileocolitis). The next most common area is the colon (Crohn's colitis). Isolated jejunal and ileal Crohn's disease also occurs.
The essential difference between ulcerative colitis and Crohn's disease is the extent of the inflammation. The inflammation of ulcerative colitis is confined to the mucosa, whereas in Crohn's disease it is transmural and is associated with fissuring ulcers and granulomas. Pseudopolyps develop in ulcerative colitis when uninvolved mucosa hypertrophies and areas of granulation tissue associated with ulcers become covered with epithelium, forming polypoid masses. 


\section{Clinical features}

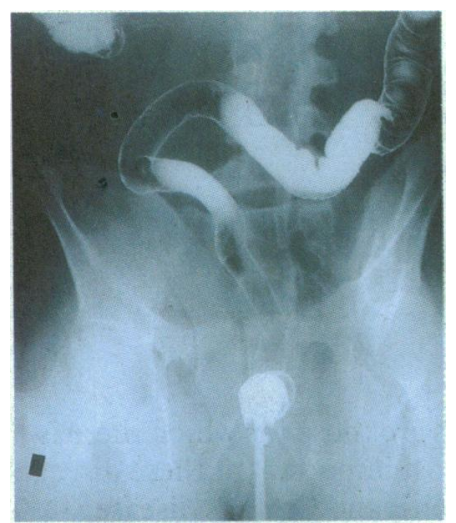

Barium enema film showing a hosepipe colon in chronic ulcerative colitis.

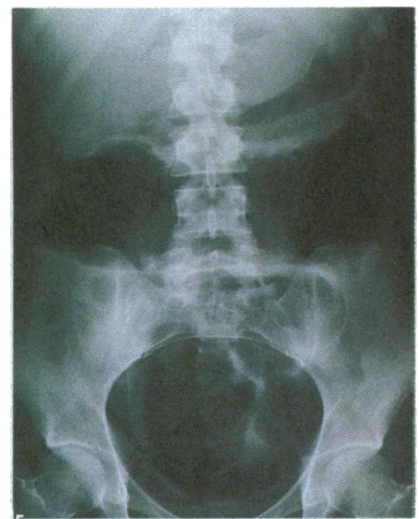

Abdominal radiograph showing toxic dilatation.

\section{Ulcerative colitis}

Ulcerative colitis may be classified clinically as mucous colitis, relapsing colitis, and toxic dilatation.

Mucous colitis is a relatively benign condition characterised by intermittent attacks of bleeding, diarrhoea, and discharge of mucus.

Relapsing colitis is progressive with similar symptoms. Radiological progression shows ulceration, pseudopolyps, and, finally, an atrophic "hosepipe" colon. It is this group of patients who are susceptible to malignant change.

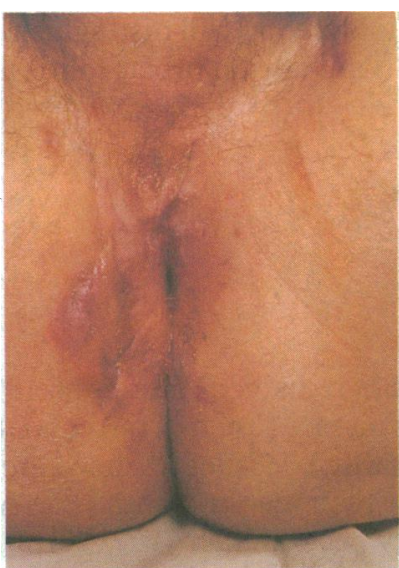

Watering can perineum.

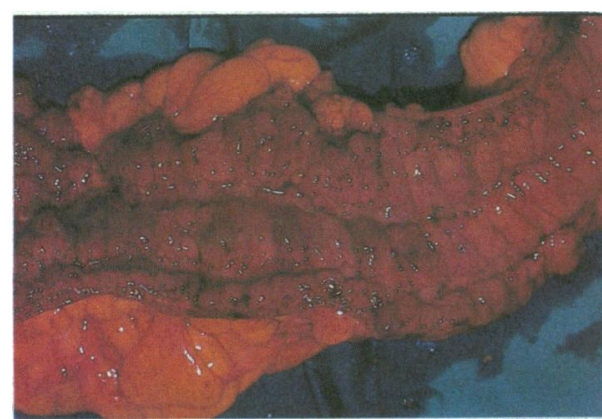

Pathological specimen of opened colon showing Crohn's colitis with deep fissuring, giving a cobblestoned appearance.

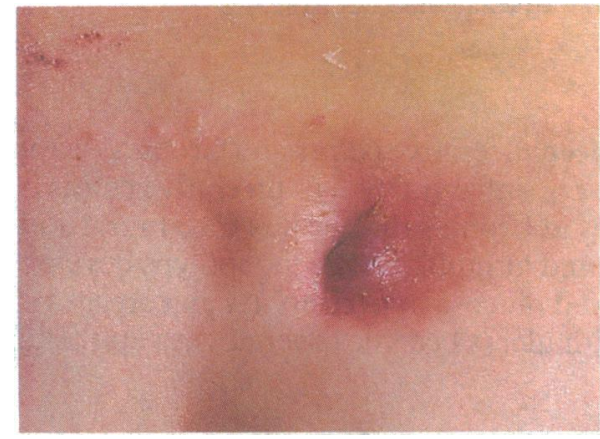

Crohn's enterocutaneous fistula emerging in lumbosacral arc.
Toxic dilatation is a life threatening condition which has to be treated by urgent colectomy. It can develop in any patient with severe colitis regardless of underlying aetiology. Signs of developing toxic dilatation are tachycardia, fever, abdominal distension, and tenderness. There is a reduction in diarrhoea as the colon becomes paralysed and abdominal radiographs show progressive colonic dilatation, particularly in the transverse colon.

\section{Crohn's colitis}

Crohn's colitis causes diarrhoea and rectal bleeding. There is commonly a history of perianal abscesses or fistulas and fleshy perianal skin tags are often present. Unlike in ulcerative colitis the rectum is commonly not affected. Ileocaecal Crohn's disease is characterised by diarrhoea caused by malabsorption of bile salts. Episodes of subacute obstruction supervene as the terminal ileum becomes narrowed and a palpable mass develops in the right iliac fossa. Less commonly ileocaecal disease presents acutely and may be confused with appendicitis. In this situation the appendix should be removed so as to avoid diagnostic confusion with future episodes of abdominal pain. Isolated jejunal or ileal Crohn's disease usually present with episodes of subacute intestinal obstruction.

\section{Fistulas}

Fistulas form in $30 \%$ of patients with Crohn's disease but are uncommon in those with ulcerative colitis. They develop because of the transmural progression of fissuring ulcers. The ulcer may erode directly into an adjacent viscus, producing an internal fistula. Alternatively a localised abscess may form, which on spontaneous or surgical drainage to the surface produces an external fistula. Most external fistulas have a low output, producing an intermittent purulent discharge. High output fistulas, producing over $500 \mathrm{ml} /$ day, usually occur because of distal obstruction. Skin excoriation is common because of the high concentration of digestive enzymes. Many internal fistulas are asymptomatic. Exceptions are enterovesical fistulas, which cause recurrent cystitis and pneumaturia, and "high-low" fistulas, which produce torrential diarrhoea. 


\section{Extraintestinal manifestations}

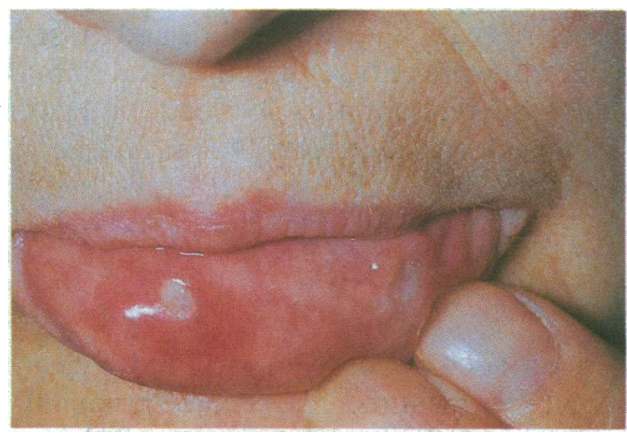

Mouth ulcers in Crohn's disease.

\section{Diagnosis}

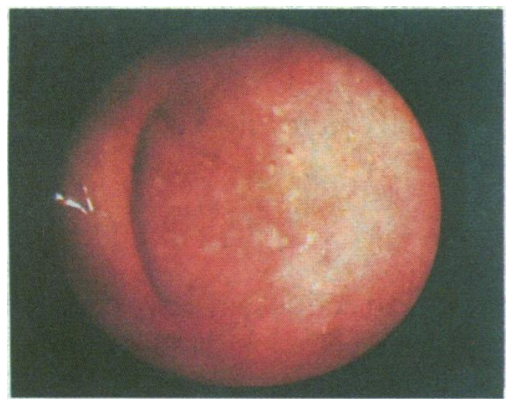

Mild ulcerative colitis viewed through a colonoscope.

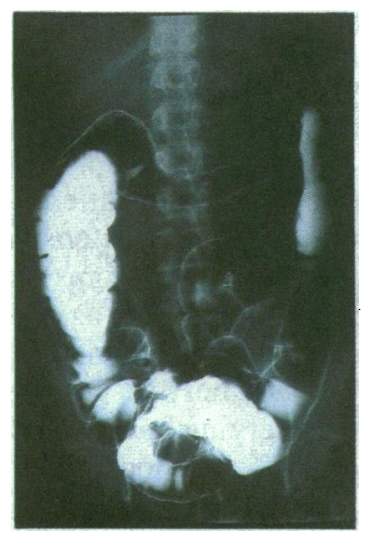

Radiograph showing segmented Crohn's colitis in transverse colon.

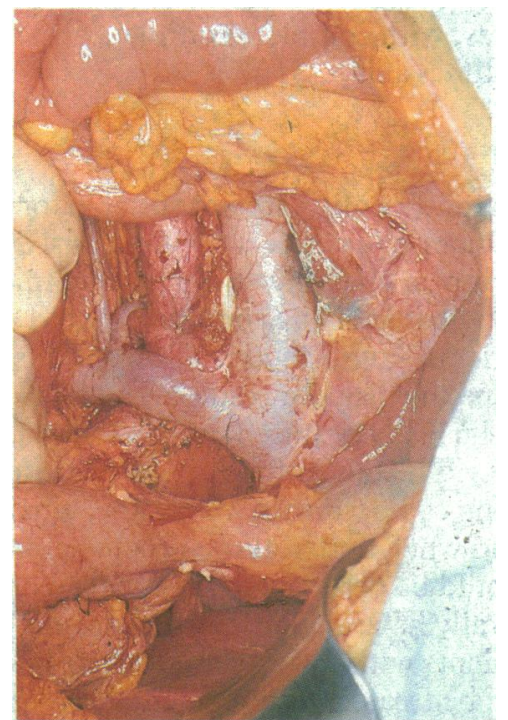

Stricture of terminal ileum in Crohn's disease.
Extraintestinal manifestations of inflammatory bowel disease occur in both ulcerative colitis and Crohn's disease. These include eye disorders, for example, episcleritis and iridocyclitis; joint disorders-for example, arthralgia, arthritis, and sacroiliitis; skin lesions - for example, erythema nodosum and pyoderma gangrenosum; aphthous mouth ulcers; finger clubbing; and sclerosing cholangitis. Ankylosing spondylitis is much commoner in patients with ulcerative colitis and Crohn's disease (1 in 100) than in the general population (1 in 10000).

Sigmoidoscopy is the most useful initial investigation in a patient presenting with diarrhoea and rectal bleeding. Other common causes of these symptoms are colorectal cancer and specific inflammatory bowel disease (infective diarrhoeas, pseudomembranous colitis, radiation enteritis, and ischaemic colitis). Sigmoidoscopy will distinguish proctocolitis from other conditions. In mild distal proctocolitis there is diffuse hyperaemia of the rectal mucosa. With more active disease there is granularity of the mucosa and contact bleeding. With severe proctocolitis a purulent exudate is seen coating the mucosa and blood oozes from its surface.

Biopsy specimens should be taken as if granulomas are present Crohn's colitis can be diagnosed. A stool sample should be sent for culture to exclude infective dysenteries. Campylobacter often produces a bloody diarrhoea and this can also occur with salmonella and shigella. Pseudomembranous colitis occurs in patients who have recently received treatment with broad spectrum antibiotics - it is caused by overgrowth of Clostridium difficile. A patchy fibrinous membrane coating the mucosa is seen on sigmoidoscopy. $C$ difficile and its toxin should be looked for on stool culture.

Barium enema examination is useful in distinguishing Crohn's colitis from ulcerative colitis and in assessing the extent and severity of colitis. Crohn's colitis is characterised by a discontinuous distribution, often with rectal sparing. Diseased segments have a cobblestoned appearance caused by deep longitudinal and transverse ulcers. "Rosethorn" ulcers may be present, which sometimes perforate to produce fistulous tracks. Mild ulcerative colitis is characterised by a disturbed mucosal pattern with small ulcers. Pseudopolyps may form in patients with more severe disease. With longstanding severe disease there is a loss of haustral markings with a featureless, shortened hosepipe colon.

Colonoscopy is also useful in assessing colitis. Although it is a more time consuming examination, biopsy specimens can be taken for histological examination. This is particularly important in the surveillance of patients with long standing and widespread ulcerative colitis as it allows severe dysplasia and early invasive cancer to be detected.

The most useful investigation in patients with suspected small bowel or ileocaecal Crohn's disease is small bowel enema examination. Small bowel Crohn's disease is characterised by the radiological "string sign," which is caused by extreme narrowing of the intestinal lumen. Fistulous tracks may also be seen.

Useful blood measurements for assessing the severity of inflammatory bowel disease and assessing the response to treatment are serum albumin concentration, which is low in patients with active disease, and erythrocyte sedimentation rate and orosomucoid and $\mathrm{C}$ reactive protein concentrations, which are raised in patients with active disease. 


\section{Treatment}
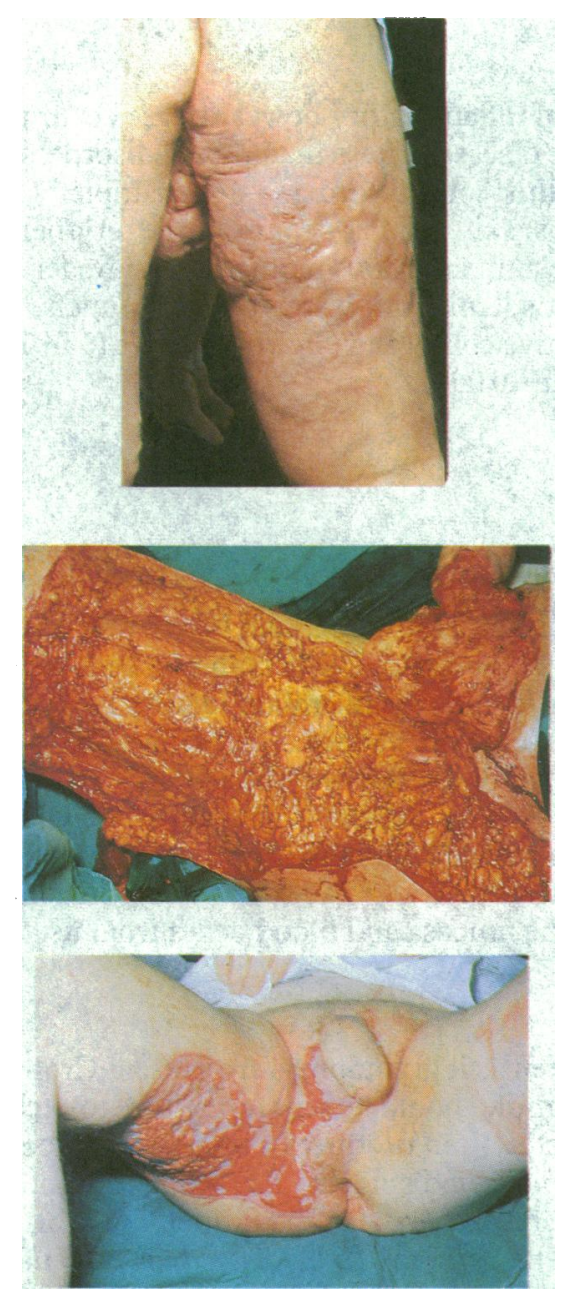

(Top) Extensive perianal Crohn's disease extending into the thigh. (Middle) The diseased skin has been excised down to the deep fascia. (Bottom) Healing perineum and thigh after skin mesh grafting.

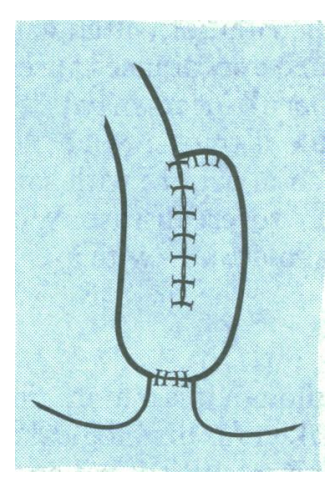

Duplicated ileoanal pouch (J Pouch). The reservoir is constructed by joining together two adjacent lengths of ileum. It is then anastomosed to the anal canal.

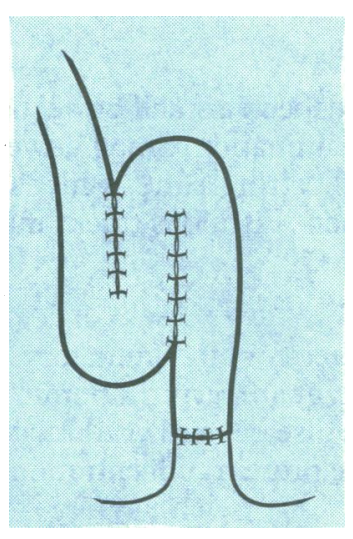

Sulphasalazine is of proved value in suppressing disease activity and maintaining remission in both patients with ulcerative colitis and those with Crohn's colitis. It should be given while patients have symptoms but may be stopped after several years of complete remission. A dose of 2-4 $\mathrm{g}$ a day is required to be effective. This can produce side effects such as nausea, vomiting, and skin rashes; slow release mesalazine is an effective alternative.

Local topical steroid preparations (such as Colifoam and Predsol enemas) are used for exacerbations of distal proctocolitis. They can be gradually tailed off as symptoms and sigmoidoscopic appearance improve. Oral prednisolone is required to control exacerbations of more widespread colitis. The dose can usually be reduced as the patients' symptoms improve. Occasionally, reduction of dosage is not possible without provoking an exacerbation of colitis, resulting in the need for long term high dosages of steroids. In these patients azathioprine can be added as an additional immunosuppressant, allowing the steroid dose to be reduced and thus reducing the risk of side effects such as osteoporosis. Azathioprine can produce marrow suppression and the blood count will need to be monitored regularly.

Patients with severe attacks of widespread colitis may have constitutional disturbances including fever, tachycardia, weight loss, and anaemia. Hospital admission is required and treatment comprises bed rest, intravenous fluids, fasting, and intravenous hydrocortisone. Patients must be carefully monitored as toxic dilatation can develop.

Indications for elective colectomy in ulcerative colitis are to restore health in patients with chronic disease, to eliminate the risk of side effects of steroids in patients requiring long term high dosages of steroids, premalignant change on colonoscopic surveillance, and patients at high risk of developing cancer (those with early onset of disease, extensive colonic involvement, and continuous rather than episodic symptoms).

Panproctocolectomy is the procedure of choice in middle aged and elderly patients. An intersphincteric approach must be used when removing the rectum to avoid damage to the pelvic autonomic nerves. Younger patients should be given the option of a sphincter preserving operation in which a pouch is constructed from a duplicated or triplicated loop of ileum.

The rectum is excised, leaving behind the anal canal with its intact sphincter mechanism. The pouch is then anastomosed to the dentate line. This gives excellent results in most cases, with complete continence and spontaneous evacuation occurring 4-6 times a day.

Patients undergoing colectomy for Crohn's colitis are not suitable for pouch formation as recurrent Crohn's disease may affect the ileum used in constructing the pouch. If the rectum appears healthy or if it is only mildly diseased such patients can be offered an ileorectal anastomosis to avoid a permanent ileostomy. One third of patients have a good result from this, one third an intermediate result, and one third a poor result because of recurrent rectal disease. Proctectomy is usually required in those with a poor result, and also in patients with florid rectal Crohn's disease causing multiple perianal fistulas - the "watering can perineum" or rectovaginal fistulas. If colonic skip lesions are found when undertaking proctectomy panproctocolectomy and ileostomy should be performed as patients with Crohn's disease with colostomies generally have a poor long term result owing to recurrent colonic disease. Patients with severe symptoms from isolated segments of Crohn's colitis can be treated with segmental colonic resection, usually with primary anastomosis. 


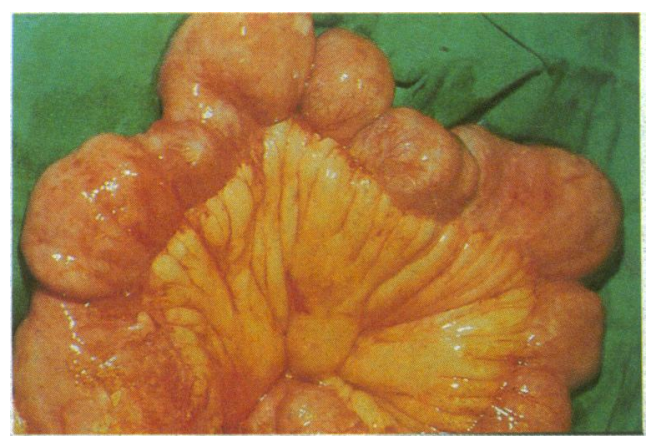

Small bowel with multiple strictures.

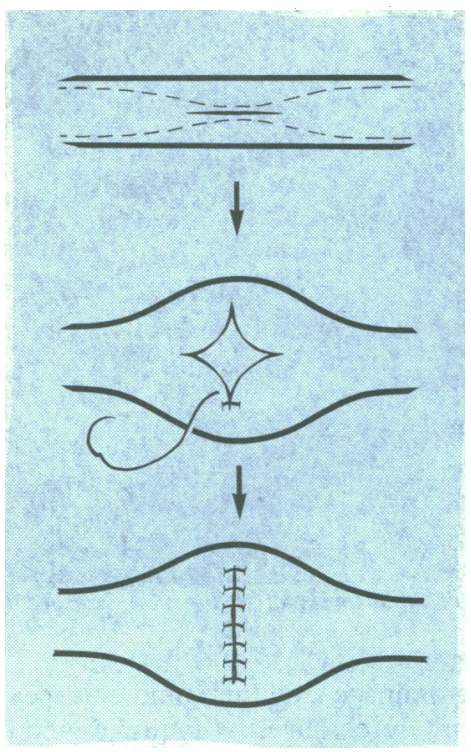

Strictureplasty. The

narrow segment of bowel

affected in Crohn's

disease is opened

longitudinally. The

opening is then closed

transversely to increase

the size of the lumen.

\section{Support for patients}

- The National Association for Colitis and Crohn's Disease is at 98a London Road, St Albans, Hertfordshire AL1 1NX

- The lleostomy Association is at Central Office, Amblehurst House, Black Scotch Lane, Mansfield, Nottinghamshire NH18 4PF
Obstructive symptoms caused by small bowel and ileocaecal Crohn's disease often respond to steroids and a low residue diet. In some patients, however, the response to steroids is poor, usually because the stricture is largely fibrous rather than inflammatory. Surgical resection is then necessary. In patients with multiple short segments of disease strictureplasty will overcome obstruction while preserving intestine. Resection of diseased small bowel and ileocaecum is also indicated in patients requiring high dosages of steroids to control their symptoms and in patients whose symptoms are not adequately controlled by medical management. Early surgery should be undertaken in those responding poorly to medical management as delay invites formation of fistulas.

Although Crohn's fistulas will occasionally respond to medical treatment with mercaptopurine, surgical resection of the diseased bowel and fistula is usually required to achieve a cure. The principle of fistula surgery is to resect the diseased bowel and fistula en bloc and to repair secondary defects in healthy intestine if at all possible. Primary anastomosis should be performed under favourable conditions, but in patients who are compromised at operation by malnutrition or sepsis, as indicated by profound hypoalbuminaemia, the two bowel ends must be exteriorised and elective reanastomosis subsequently undertaken.

Patients with inflammatory bowel disease often need a great deal of support in coping with their disease, especially if they are to undergo surgery with stoma formation. The National Association for Colitis and Crohn's Disease (NACC) is a useful organisation for such patients. It produces helpful booklets and newsletters and holds regular meetings for members.

The photographs were produced by the department of medical illustration, Salford Health Authority, and we thank Dr Roberts and Dr Sheridan, Hope Hospital, for providing the histology slides and radiographs, respectively. The line drawings were prepared by Paul Somerset, medical illustration department, Wythenshawe Hospital.

Mr S Pettit is consultant surgeon, Victoria Hospital, Blackpool, and Professor M H Irving is professor of general surgery, Hope Hospital, Salford.

The ABC of Colorectal Diseases has been edited by Mr D J Jones, lecturer and honorary senior registrar, and Professor M H Irving, Hope Hospital, Salford. 